Published in final edited form as:

Cancer Causes Control. 2013 January ; 24(1): 13-25. doi:10.1007/s10552-012-0078-8.

\title{
Diabetes and risk of pancreatic cancer: a pooled analysis from the pancreatic cancer cohort consortium
}

\author{
Joanne W. Elena,

\section{Emily Steplowski,} \\ Information Management Services, Silver Spring, MD, USA
}

Division of Cancer Control and Population Science (DCCPS), National Cancer Institute, National Institutes of Health, Department of Health and Human Services, Bethesda, MD, USA

\section{Kai Yu,}

Division of Cancer Epidemiology and Genetics, National Cancer Institute, National Institutes of Health, Department of Health and Human Services, Bethesda, MD, USA

\section{Patricia Hartge, \\ Division of Cancer Epidemiology and Genetics, National Cancer Institute, National Institutes of Health, Department of Health and Human Services, Bethesda, MD, USA}

\section{Geoffrey S. Tobias,}

Division of Cancer Epidemiology and Genetics, National Cancer Institute, National Institutes of Health, Department of Health and Human Services, Bethesda, MD, USA

\section{Michelle J. Brotzman, \\ Westat, Inc, Rockville, MD, USA}

\section{Stephen J. Chanock,}

Division of Cancer Epidemiology and Genetics, National Cancer Institute, National Institutes of Health, Department of Health and Human Services, Bethesda, MD, USA

Laboratory of Translation Genomics, Division of Cancer Epidemiology and Genetics, National Cancer Institute, National Institutes of Health, Department of Health and Human Services, Bethesda, MD, USA

\section{Rachael Z. Stolzenberg-Solomon,}

Division of Cancer Epidemiology and Genetics, National Cancer Institute, National Institutes of Health, Department of Health and Human Services, Bethesda, MD, USA

\section{Alan A. Arslan,}

Department of Obstetrics and Gynecology, New York University School of Medicine, New York, NY, USA

\footnotetext{
(c) Springer Science+Business Media Dordrecht (outside the USA) 2012

Correspondence to: Joanne W. Elena, elenajw@mail.nih.gov.

Laufey Amundadottir, Alan A. Arslan, H. Bas Bueno-de-Mesquita, Eric J. Duell, Charles S. Fuchs, Myron Gross, Kathy Helzlsouer, Eric J. Jacobs, Charles Kooperberg, Andrea LaCroix, Gloria Petersen, Rachael Z. Stolzenberg-Solomon, Kala Visvanathan, Brian M. Wolpin, Wei Zheng: Steering committee members.

Present Address: L. Sansbury, Department of Epidemiology, GlaxoSmithKline, Research Triangle Park, NC, USA

M. S. Simon, Karmanos Cancer Institute, Department of Oncology, Wayne State University, Detroit, MI, USA

N. Slimani, International Agency for Research on Cancer, Lyon, France

D. Trichopoulos, Bureau of Epidemiologic Research, Academy of Athens, Athens, Greece

J. Virtamo, Department of Chronic Disease Prevention, National Institute for Health and Welfare, Helsinki, Finland

B. M. Wolpin - C. S. Fuchs, Department of Medical Oncology, Dana-Farber Cancer Institute, Boston, MA, USA

M. Gross, Department of Laboratory Medicine/Pathology, School of Medicine, University of Minnesota, Minneapolis, MN, USA
} 
Department of Environmental Medicine, New York University School of Medicine, New York, NY, USA

New York University Cancer Institute, New York, NY, USA

H. Bas Bueno-de-Mesquita,

National Institute for Public Health and the Environment (RIVM), Bilthoven, The Netherlands

Department of Gastroenterology and Hepatology, University Medical Center Utrecht, Utrecht, The Netherlands

Kathy Helzlsouer,

Prevention and Research Center, Mercy Medical Center, Baltimore, MD, USA

Eric J. Jacobs,

Epidemiology Research Program, American Cancer Society, Atlanta, GA, USA

Andrea LaCroix,

Fred Hutchinson Cancer Research Center, Seattle, WA, USA

Gloria Petersen,

Department of Health Sciences Research, Mayo Clinic, Rochester, MN, USA

Wei Zheng,

Division of Epidemiology, Department of Medicine, Vanderbilt Epidemiology Center, and

Vanderbilt-Ingram Cancer Center, Vanderbilt University, Nashville, TN, USA

\section{Demetrius Albanes,}

Division of Cancer Epidemiology and Genetics, National Cancer Institute, National Institutes of Health, Department of Health and Human Services, Bethesda, MD, USA

Naomi E. Allen,

Cancer Epidemiology Unit, University of Oxford, Nuffield Department of Clinical Medicine, Oxford, UK

\section{Laufey Amundadottir,}

Division of Cancer Epidemiology and Genetics, National Cancer Institute, National Institutes of Health, Department of Health and Human Services, Bethesda, MD, USA

Laboratory of Translation Genomics, Division of Cancer Epidemiology and Genetics, National Cancer Institute, National Institutes of Health, Department of Health and Human Services, Bethesda, MD, USA

\section{Ying Bao,}

Channing Laboratory, Department of Medicine, Brigham and Women's Hospital and Harvard Medical School, Boston, MA, USA

Heiner Boeing,

German Institute of Human Nutrition Potsdam-Rehbrüecke, Nuthetal, Germany

Marie-Christine Boutron-Ruault,

Inserm, (Institut National de la Santé et de la Recherche Médicale) and Institut Gustave Roussy, Villejuif, France

\section{Julie E. Buring,}

Divisions of Preventive Medicine and Aging, Department of Medicine, Brigham and Women's Hospital and Harvard Medical School, Boston, MA, USA

Department of Ambulatory Care and Prevention, Harvard Medical School, Boston, MA, USA

\section{J. Michael Gaziano,}


Physicians' Health Study, Divisions of Aging, Cardiovascular Medicine, and Preventive Medicine, Department of Medicine, Brigham and Women's Hospital and Harvard Medical School, Boston, MA, USA

Massachusetts Veterans Epidemiology Research and Information Center, Veterans Affairs Boston Healthcare System, Boston, MA, USA

Edward L. Giovannucci, Department of Epidemiology, Harvard School of Public Health, Boston, MA, USA

Department of Biostatistics, Harvard School of Public Health, Boston, MA, USA

Department of Nutrition, Harvard School of Public Health, Boston, MA, USA

Eric J. Duell,

Unit of Nutrition, Environment and Cancer, Catalan Institute of Oncology (ICO-IDIBELL),

Barcelona, Spain

Göran Hallmans,

Department of Public Health and Clinical Medicine, Nutritional Research, Umeå University, Umeå, Sweden

Barbara V. Howard,

MedStar Health Research Institute, Georgetown University, Hyattsville, MD, USA

David J. Hunter,

Department of Epidemiology, Harvard School of Public Health, Boston, MA, USA

Department of Biostatistics, Harvard School of Public Health, Boston, MA, USA

\section{Amy Hutchinson,}

Laboratory of Translation Genomics, Division of Cancer Epidemiology and Genetics, National

Cancer Institute, National Institutes of Health, Department of Health and Human Services,

Bethesda, MD, USA

Core Genotyping Facility, SAIC-Frederick Inc., NCI-Frederick, Frederick, MD, USA

Kevin B. Jacobs,

Division of Cancer Epidemiology and Genetics, National Cancer Institute, National Institutes of Health, Department of Health and Human Services, Bethesda, MD, USA

\section{Charles Kooperberg,}

Fred Hutchinson Cancer Research Center, Seattle, WA, USA

\section{Peter Kraft,}

Department of Epidemiology, Harvard School of Public Health, Boston, MA, USA

Julie B. Mendelsohn,

Division of Cancer Epidemiology and Genetics, National Cancer Institute, National Institutes of Health, Department of Health and Human Services, Bethesda, MD, USA

Dominique S. Michaud,

Division of Biology and Medicine, Brown University, Providence, RI, USA

Division of Epidemiology, Public Health and Primary Care, Imperial College, London, UK

Domenico Palli,

Molecular and Nutritional Epidemiology Unit, Cancer Research and Prevention Institute-ISPO, Florence, Italy

Lawrence S. Phillips,

Division of Endocrinology, Emory University, Atlanta, GA, USA 
Atlanta VA Medical Center, Decatur, GA, USA

Kim Overvad,

Department of Epidemiology, School of Public Health, Aarhus University, Aalborg, Denmark

Alpa V. Patel, Epidemiology Research Program, American Cancer Society, Atlanta, GA, USA

Leah Sansbury,

Division of Cancer Control and Population Science (DCCPS), National Cancer Institute, National Institutes of Health, Department of Health and Human Services, Bethesda, MD, USA

Xiao-Ou Shu, Division of Epidemiology, Department of Medicine, Vanderbilt Epidemiology Center, and Vanderbilt-Ingram Cancer Center, Vanderbilt University, Nashville, TN, USA

Michael S. Simon, Nadia Slimani, Dimitrios Trichopoulos, Department of Epidemiology, Harvard School of Public Health, Boston, MA, USA

Kala Visvanathan, Department of Epidemiology, Johns Hopkins Bloomberg School of Public Health, Baltimore, MD, USA

Johns Hopkins Sidney Kimmel Comprehensive Cancer Center, Baltimore, MD, USA

Jarmo Virtamo, Brian M. Wolpin, Channing Laboratory, Department of Medicine, Brigham and Women's Hospital and Harvard Medical School, Boston, MA, USA

Anne Zeleniuch-Jacquotte, Department of Environmental Medicine, New York University School of Medicine, New York, NY, USA

New York University Cancer Institute, New York, NY, USA

Charles S. Fuchs, Channing Laboratory, Department of Medicine, Brigham and Women's Hospital and Harvard Medical School, Boston, MA, USA

Robert N. Hoover, and Division of Cancer Epidemiology and Genetics, National Cancer Institute, National Institutes of Health, Department of Health and Human Services, Bethesda, MD, USA

\section{Myron Gross}

Joanne W. Elena: elenajw@mail.nih.gov

\section{Abstract}

Purpose-Diabetes is a suspected risk factor for pancreatic cancer, but questions remain about whether it is a risk factor or a result of the disease. This study prospectively examined the association between diabetes and the risk of pancreatic adenocarcinoma in pooled data from the NCI pancreatic cancer cohort consortium (PanScan).

Methods-The pooled data included 1,621 pancreatic adenocarcinoma cases and 1,719 matched controls from twelve cohorts using a nested case-control study design. Subjects who were diagnosed with diabetes near the time ( $<2$ years) of pancreatic cancer diagnosis were excluded from all analyses. All analyses were adjusted for age, race, gender, study, alcohol use, smoking, $\mathrm{BMI}$, and family history of pancreatic cancer. 
Results-Self-reported diabetes was associated with a forty percent increased risk of pancreatic cancer $(\mathrm{OR}=1.40,95 \% \mathrm{CI}: 1.07,1.84)$. The association differed by duration of diabetes; risk was highest for those with a duration of $2-8$ years $(\mathrm{OR}=1.79,95 \% \mathrm{CI}: 1.25,2.55)$; there was no association for those with $9+$ years of diabetes $(\mathrm{OR}=1.02,95 \% \mathrm{CI}: 0.68,1.52)$.

Conclusions-These findings provide support for a relationship between diabetes and pancreatic cancer risk. The absence of association in those with the longest duration of diabetes may reflect hypoinsulinemia and warrants further investigation.

\section{Keywords}

Diabetes; Risk factor; Cohort consortium; Pancreatic cancer

\section{Introduction}

Pancreatic adenocarcinoma is the fourth leading cause of cancer death in the United States [1] and annually accounts for more than 227,000 deaths worldwide [2]. It is a highly fatal disease with a five-year survival rate of less than $5 \%$ [1]. Several risk factors have been identified including age, smoking, race, sex, diabetes, alcohol intake, ABO blood group, body mass index (BMI), and family history of pancreatic cancer [3-9]. Pancreatic cancer risk is higher in Blacks than Whites, men than women, obese than normal weight, and diabetics compared to non-diabetics [3]. Smoking is the most studied and well-characterized risk factor; other possible risk factors are less established $[3,4,10]$.

Type 2 diabetes, generally discovered during adulthood, is usually marked by an initial period of relative hyperinsulinemia, in which the mass of the $\beta$-cells of the pancreatic islets of Langerhans is reduced but insulin secretion increased as partial compensation for increased insulin resistance due to overweight and lack of physical activity, followed later by a period of relative hypoinsulinemia, reflecting further reduction of the mass of the $\beta$ cells [11]. Hyperglycemia is associated with altered glucose metabolism, chronic inflammation, oxidative stress, and the activation of insulin signaling cascades that may increase the risk of pancreatic cancer [12]. Diabetes can also be an early manifestation of pancreatic cancer, so it is important to determine whether diabetes is an independent risk factor for pancreatic cancer or instead a consequence of the disease. Pancreatic cancer progresses without significant early symptoms and is generally diagnosed at late stages. The development of diabetes within a few years of a pancreatic cancer diagnosis is more likely to suggest an effect of the tumor, whereas diabetes of longer duration is more likely to contribute to the development of cancer [13].

Two meta-analyses concluded that diabetes is associated with an increased risk of pancreatic cancer $[14,15]$; however, many of the studies included in these analyses were limited by low numbers of pancreatic cancer cases, were likely to involve secondary effects of pancreatic cancer including the induction of diabetes, and had limited numbers of prospective studies with an evaluation of the long-term effects of diabetes. In addition, these studies generally did not evaluate the effect of diabetes duration. Thus, additional investigations with adequate numbers of cases in prospective studies are needed to discern this complicated relationship, especially in light of the increasing prevalence of diabetes in many Western countries.

This report examines the association between type 2 diabetes and subsequent pancreatic adenocarcinoma risk by pooling data from 12 cohort studies in the NCI Pancreatic Cancer Cohort Consortium (PanScan) using a nested case-control study design. These cohorts have long-term follow-up and include 1,621 pancreatic adenocarcinoma cases, making this one of the largest prospective analyses to date of type 2 diabetes and pancreatic cancer. 


\section{Methods}

\section{Study population}

PanScan is an initiative that was funded jointly by the National Cancer Institute's Division of Cancer Control and Population Sciences and the Division of Cancer Epidemiology and Genetics in 2006. PanScan includes investigators from 12 prospective epidemiologic cohorts and one case-control study, the Mayo Clinic Study. It was created to investigate environmental, lifestyle, and genetic causes of pancreatic cancer. Studies in the pooled analysis included: The Alpha-Tocopherol, Beta-Carotene Cancer Prevention Trial (ATBC) [16], CLUE II [17], Cancer Prevention Study II (CPS II) [18], European Prospective Investigation into Cancer and Nutrition (EPIC) [19], the Health Professionals Follow-up Study (HPFS) [20], the Mayo Clinic study (MAYO) [21], the New York University Women's Health Study (NYUWHS) [22], the Nurses' Health Study (NHS) [20], the Physicians' Health Study (PHS I) [20], the Prostate, Lung, Colorectal, Ovarian Cancer Screening Trial (PLCO) [23], Shanghai Men's and Women's Health Studies (SMWHS) [24, 25], the Women's Health Initiative (WHI) [26], and the Women's Health Study (WHS) [27]. Characteristics of these studies have been summarized previously including the years of recruitment, age range of participants, racial composition, years of follow-up, and matching criteria [16-27]. The Mayo study was excluded from these analyses due to its case-control study design. A total of 120 cases and 79 controls had missing data on diabetes and thus were excluded from analysis. We have also excluded participants diagnosed with diabetes less than 2 years before pancreatic cancer diagnosis or selection as a control $(n=36)$ and also any participants diagnosed with diabetes after cancer/selection $(n=4)$ in our analyses. A total of 1,621 cases and 1,719 controls were included in the current analysis.

\section{Case ascertainment and data collection}

Cases included all incident primary pancreatic adenocarcinoma (ICD-O-3 codes C25.0C25.3, C25.7-C25.9). Endocrine pancreatic tumors (ICD-O-3 code C25.4, histology types $8,150,8,151,8,153,8,155,8,240$, and 8,246$)$ were excluded because the etiology of these cancers is thought to be different from that of exocrine tumors, which account for the vast majority of pancreatic tumors. Case ascertainment varied between studies but included linking participants to cancer registries and national death indices, and using self and next of kin reports. Most cases were histologically confirmed (ATBC, CLUE II, EPIC, NYUWHS, SMWHS, WHI) or confirmed through cancer registries (ATBC, CPS II, EPIC, SMWHS), death certificates (CPS II, EPIC), or review of medical records by medical personnel (ATBC, CPS II, EPIC, NHS, PHS I, PLCO, SMWHS).

Controls were incidence density-sampled with a 1-to-1 control-to-case ratio and were alive and cancer-free on the date of diagnosis of the matched case. At a minimum, controls were matched to cases by calendar year of birth ( \pm 5 years), gender, race and ethnicity. Some cohorts employed more stringent matching on age such as age at baseline or age at blood draw ( \pm 5 years), and additionally, on other relevant factors such as smoking, date/time of day of blood draw, fasting blood draw (for comparisons of blood levels of analytes of interest), and length of follow-up. Detailed descriptions of data collection methods have been published previously by the individual studies [16-27]. Baseline information on BMI, waist circumference, waist-to-hip ratio, history of cigarette smoking, gender, age, race, family history of pancreatic cancer in a first-degree relative, alcohol consumption, pancreatitis, and history of diabetes was requested from the cohorts that collected this information. Data on diabetes, demographics, and possible confounders were collected through self-administered written questionnaires or in-person interviews from baseline and/ or follow-up questionnaires, with the most recent data available used in analysis. Selfreported diabetes status was ascertained based on a physician's diagnosis of diabetes and/or 
treatment for diabetes with the use of insulin or oral hypoglycemic drugs. The duration of diabetes was assessed by the reported age at diagnosis, when available, and the reported follow-up period. When the age of diagnosis was not available, it was estimated from the date of the follow-up examination that first reported the presence of diabetes. Three cohorts (ATBC, PLCO, and WHI) did not query age at diabetes diagnosis; these data were missing for $22 \%$ of participants in the remaining cohorts. Individual datasets were checked for consistency with previously published results. The Special Studies Institutional Review Board (SSIRB) of the National Cancer Institute approved the pooled PanScan study. Each study also was approved by its local IRB.

\section{Statistical analysis}

Odds ratios (ORs) and $95 \%$ confidence intervals (95\% CIs) for pancreatic cancer risk were calculated using unconditional logistic regression. Several multivariate models were used to assess the effects of potential confounders. Unless otherwise noted, models were adjusted for age ( $<=50,51-60,61-70,71-80$, and $81+$ years), gender, race (European, African, Asian, other, unknown), study, continuous BMI, alcohol use (never, ever current status unknown, former drinker, light current drinker, heavy current drinker, unknown), smoking (never, former, current, unknown), and family history of pancreatic cancer. Indicator variables were used in all analysis for variables with missing responses. No adjustment was made for pancreatitis because few cohorts had this information. Several approaches, including visual representation and splines, were used to evaluate the duration of diabetes when modeling the effect of diabetes on pancreatic cancer risk but did not change results appreciably. We used cut points of $2-8$ years and nine or more years based on the numbers of responses to ensure adequate cell size for analysis.

We modeled multiplicative interaction terms between diabetes and gender, age, cigarette smoking status (never, former, current), and continuous BMI and compared $p$ values for the likelihood ratio tests $(<0.05)$ for the models with and without interaction terms. We also evaluated heterogeneity across studies by fitting models with and without multiplicative interaction terms between diabetes and study and calculating a $p$ value for heterogeneity using the likelihood ratio statistic. All analyses were conducted using SAS version 9.1.3 (SAS Institute Inc, Cary, North Carolina).

\section{Results}

Table 1 describes each study in the PanScan study included in these analyses, including the study location, numbers of participants, age range, method to assess diabetes status, and the prevalence of diabetes among cases and controls, while Table 2 presents the baseline characteristics of the participants included. There were approximately equal numbers by age, gender, and race among cases and controls. There were small numbers of Asians and Africans, and most of the participants were between the ages of 60 and 80 . Cases were more likely to identify as current smokers than controls ( 28 vs. $22 \%$ ) and were more likely to be overweight or obese. Cases were more likely to report being diagnosed with diabetes than controls (10 vs. $7 \%$ ). A history of pancreatitis and/or family history of pancreatic cancer were slightly more common among cases as compared to controls. However, many cohorts did not collect this information and approximately one-half of the participants lacked these data. The mean age at pancreatic adenocarcinoma diagnosis was 68.9 years (SD 8.5), whereas the mean age at diabetes diagnosis was 58.5 years (SD 12.4).

Diabetes was associated with an increased risk of pancreatic cancer (Odds ratio $(\mathrm{OR})=1.37$, $95 \%$ CI: 1.06, 1.77); adjustment for age, gender, study, and race had a minor effect on the odds ratio ( $\mathrm{OR}=1.49,95 \% \mathrm{CI}: 1.14,1.94)$, as did adjustment for the full model including age, race, gender, study, alcohol use, smoking, BMI, and family history of pancreatic cancer 
$(\mathrm{OR}=1.40,95 \% \mathrm{CI}: 1.07,1.84)$. Because a consequence of pancreatic cancer can be the development of diabetes, all analyses excluded pancreatic cancer cases with diabetes diagnosed within 2 years of the pancreatic cancer diagnosis, as well as controls diagnosed with diabetes within 2 years of the diagnosis date of the matched case. When those cases diagnosed/controls enrolling within 2 years of a diabetes diagnosis were included, the odds ratio was modestly higher $(\mathrm{OR}=1.50,95 \% \mathrm{CI}: 1.18,1.91)$.

We also examined whether the duration of diabetes was associated with the risk of pancreatic adenocarcinoma (Table 3). Due to the limited number of cases for some time periods, several time periods were grouped together to ensure adequate numbers of cases for analysis. The optimal groupings were cases with a duration of 2-8 years and 9+ years of duration. Changing the periods of duration within the grouping yielded similar results (Table 4). The risk of pancreatic adenocarcinoma was elevated when the duration of diabetes ranged from $2-8$ years $(\mathrm{OR}=1.79,95 \% \mathrm{CI}$ : $1.25,2.55)$. Interestingly, the risk of pancreatic adenocarcinoma decreased with a duration of diabetes of $9+$ years $(\mathrm{OR}=1.02,95 \%$ CI: $0.68,1.52)$ as compared to a duration of diabetes from $2-8$ years. Similar results were found for men $(\mathrm{OR}=1.79,95 \% \mathrm{CI}: 1.00,3.21)$ and women (OR = 1.81, $95 \% \mathrm{CI}: 1.15,2.87)$ for those with a duration of diabetes for 2-8 years. In a fully adjusted model, we adjusted for the potential confounders of age, gender, race, study, BMI, alcohol use, smoking, and family history of pancreatic cancer. Examination of possible effect modification with BMI, age, and smoking did not reveal any statistically significant interactions. In addition, we tested for heterogeneity among the studies included in these analyses using Cochran's $Q$ test and found no evidence of heterogeneity ( $p=0.317$ for $2-8$ years and $p=0.923$ for $9+$ years duration).

The ORs for each of the 12 cohorts are shown in a Forest plot for those with the duration of diabetes of 2-8 years (Fig. 1) and nine or more years (Fig. 2). Four cohorts (HPFS, PHS, WHS, and NYU) were not included in the summary estimates and are not displayed because these cohorts contained too few cases with diabetes that matched these criteria to calculate meaningful estimates. For diabetes duration of 2-8 years, the ORs are consistent across the cohorts indicating a positive association of diabetes with pancreatic cancer, with a range of ORs from 1.06 to 12.0 with the exception of NHS (OR $=0.43,95 \%$ CI: $0.04,5.07)$. The risks seen in Fig. 1 are attenuated for each cohort in Fig. 2, with risk estimates for most studies being approximately null. The wide confidence limits seen were a consequence of low case numbers in many cohorts.

\section{Discussion}

The purpose of the current study was to examine the association between diabetes and pancreatic adenocarcinoma risk by pooling data from nested case-control studies included in the NCI Pancreatic Cancer Cohort Consortium (PanScan). Diabetes appears to be a moderate risk factor for pancreatic cancer risk with $40 \%$ higher risk seen in diabetics than non-diabetics. Those with a duration of diabetes of $2-8$ years were at the highest risk, being 1.8 times as likely to develop pancreatic cancer as non-diabetics. No increased risk was observed for those with the longest duration of diabetes ( $>9$ years). The association between diabetes and pancreatic adenocarcinoma was not modified by gender, smoking, age, or BMI.

Diabetes was established as a possible risk factor for pancreatic cancer based primarily on two well-conducted meta-analyses $[14,15]$ that produced summary estimates that are very similar to ours. The first meta-analysis of nine cohort studies and 11 case-control studies reported a pooled relative risk (RR) of 2.1 and found higher risk in the cohort studies (RR: 2.6) than the case-control studies (RR: 1.8) [14]. A subsequent meta-analysis of 19 cohort studies and 17 case-control studies also found slightly lower risk for cohort studies (RR: 
1.73) than the case-control studies (RR: 1.94), with an overall summary OR of 1.82[15]. Both meta-analyses excluded cases diagnosed with diabetes within 1 year of pancreatic cancer, which could produce slightly higher risk estimates than those provided here excluding those with a duration of diabetes $<2$ years. However, our risk estimates were quite similar (1.79 vs. 2.1 and 1.71). Since the publication of these meta-analyses, additional epidemiologic studies have been conducted. The vast majority have reported similar positive associations [28-31], with ORs ranging from 1.8 to 3.2. One population-based cohort of approximately 18,000 men observed a nonsignificant association, of diabetes with pancreatic cancer mortality (RR: 2.47, $95 \%$ CI: 0.79, 7.75), but was limited by low numbers of cases [32]. Another study of national hospital and cancer registries for type 2 diabetics hospitalized in Sweden with up to 40 years of follow-up observed elevated standardized incidence ratios (SIR), which were standardized to the general Swedish population, for pancreatic cancer risk, with SIRs of 6.08 overall, 3.57 excluding the first year of follow-up, and 1.80 excluding the first 5 years of follow-up [33]. Nonetheless, interpretation of the association between diabetes and pancreatic cancer has been complicated by the difficulty of developing large prospective studies with sufficient numbers of cases and follow-up periods. Many of these studies have included small numbers of cases with limited follow-up information.

In contrast to our results, an increased risk of pancreatic cancer for those with the greatest duration of diabetes was observed in the two meta-analyses discussed above. For example, Huxley et al. [15] reported a slight attenuation of risk with duration $>5$ years, compared to 1-4 years, yet saw elevated risk with diabetes regardless of duration (RR: 2.05 for duration 1-4 years, RR: 1.54 for duration 5-9 years and RR: 1.51 duration for duration > 10 years). One might expect a longer exposure to diabetes to be associated with higher cancer risk; however, type 2 diabetes begins with insulin resistance and relative hyperinsulinemia. Later in the natural history, there is further loss of the islet $\beta$-cells, resulting in hypoinsulinemia [34]. If insulin levels contribute to diabetes and act as an independent risk factor for pancreatic cancer, then it follows that patients with a longer duration of diabetes, who are more likely to be hypoinsulinemic, would be at lower risk compared to those who are earlier in their diabetes natural history and have higher serum insulin levels. In fact, biomarker studies have demonstrated greater risk of pancreatic cancer with prediagnostic elevations in post-load plasma glucose [35, 36], C-peptide in nonfasting blood specimens [37], serum and plasma glucose [38, 39], HbA1c [40], and insulin [41]. Furthermore, the diabetes drug metformin, an insulin sensitizer which tends to lower insulin levels, has been associated with decreased pancreatic risk, whereas insulin and insulin secretagogues have been associated with increased pancreatic cancer risk, [42, 43] offering additional support to the hypothesis that insulin is the agent affecting risk. However, it should be recognized that many diabetics receive insulin injections, and it is possible that the insulin levels resulting from the administered insulin would also contribute to risk of pancreatic cancer. Studies of type 1 diabetics who receive routine insulin treatment have not demonstrated an excess risk of pancreatic cancer, albeit these studies are limited by very low numbers [42, 43]. It is possible that many of the type 1 diabetics were relatively hypoinsulinemic even after the insulin injections, since type 1 diabetes patients are generally less insulin-resistant and relatively hypoinsulinemic compared to type 2 diabetes patients. The lack of an effect of administered insulin may also be the result of differences in the sites of action due to differences in the site of production as compared with routes of administration. Alternatively, there may be additional biological abnormalities beyond insulin and the IGF pathway involved in diabetes (e.g., C-peptide or elevated circulating free fatty acids) that influence pancreatic cancer risk.

The relationship between diabetes and pancreatic cancer risk is further complicated by the potential for pancreatic cancer to cause diabetes [34]. Thus, it is vital in studies of risk to 
exclude any patient in whom diabetes has been caused by pancreatic cancer. Although there are no clinical features to distinguish diabetes due to pancreatic cancer from other diabetes, a short duration between diabetes diagnosis and pancreatic cancer diagnosis suggests that diabetes may result from pancreatic cancer [13, 44]. A recent case-control study of pancreatic cancer cases $(n=736)$ and age- and sex-matched controls $(n=1,875)$ evaluated the temporality of the relationship using clinical information of fasting glucose levels collected up to 5 years before diagnosis of pancreatic cancer [13]. The proportion of cases with diabetes and controls with diabetes was similar in the time period 3-5 years before pancreatic cancer diagnosis, with a small increase in the proportion of diabetic cases reported 3 years before diagnosis, and a large increase in the proportion of diabetic cases within 2 years of a pancreatic cancer diagnosis. These results suggest that diabetes discovered during the 2 years prior to pancreatic cancer diagnosis is likely the result of the cancer. In an effort to minimize the effect of possible reverse causality on our results, we excluded individuals diagnosed with diabetes within 2 years of their pancreatic cancer diagnosis. This type of reverse causality is less of a concern among those with a longer duration because pancreatic cancer is a highly fatal disease and it is unlikely that someone with undetected pancreatic cancer would remain healthy for a period of many years. A recent study on the natural history of pancreatic cancer found that almost 20 years span from the initiating mutation until cancer death; with 11.7 years from mutation to the rise of a parental clone, another 6.8 years until the development of metastatic subclones, and 2.7 years until death, on average [45]. Interpreting our findings that diabetes is a risk factor for those with diabetes for 2-8 years, but not longer than 9 years, before pancreatic cancer diagnosis, in light of this analysis suggests that the effect of diabetes occurs during the phase where the parental clone leads to the development of metastatic subclones and their escape from the pancreas.

Obesity is the leading cause of type 2 diabetes. Both obesity and diabetes appear to be risk factors of pancreatic cancer but it is difficult to fully tease apart their independent effects. Many of the hypothesized biologic pathways are the same for diabetes and obesity including insulin resistance, inflammation, and oxidative stress [12,35]. Although the results have been mixed, most studies have found that higher BMI is correlated with an increased risk of pancreatic cancer [12, 46], as well as younger age at onset and poorer survival [47]. A recent study of the same pooled data used here found elevated risk for those in the highest quartile of BMI (OR $=1.33,95 \% \mathrm{CI}: 1.12,1.58)$ [9], as did a similar study of 14 cohorts with $>2,000$ pancreatic cancer cases which found the obese to be at $54 \%$ higher risk [48]. Our associations with diabetes remained after adjustment for BMI, as did the BMI associations when adjusted for diabetes, suggesting that they may have separate effects on risk.

This study had a number of strengths including a large number of cases with considerable follow-up. The prospective nature of the cohorts allowed diabetes status to be assessed before the diagnosis of cancer, eliminating recall bias. By excluding patients diagnosed with diabetes within 2 years of a pancreatic cancer diagnosis, we minimized the possibility that associations seen here are due to reverse causality. The major limitation is that we measured diabetes from self-report in cohorts of people who were enrolled before the recent rise in diabetes. Recent estimates of the prevalence of diabetes in the United States among those over 20 years of age are approximately $11 \%$ [49]. Most of the cohorts included in this analysis had much lower prevalence, with the exception of CLUE II, in large part because they occurred decades earlier. Further, approximately one-third of diabetics are estimated to be undiagnosed [50]. The resulting misclassification would be expected to be nondifferential across cases and controls and would tend to bias our results toward the null. In addition, self-reported estimations of duration may not be accurate and we were unable to include glucose or insulin measures in the models. There is also the possibility of misclassification of pancreatic cancer cases; however, cases were rigorously confirmed and 
it is unlikely that subjects would remain healthy with asymptomatic pancreatic cancer for years. Due to very low numbers of people reporting type 1 diabetes in our sample $(n=4$ among cases), we did not analyze type 1 and type 2 diabetes separately and thus cannot assess whether the association with pancreatic cancer is different. We did not have available medication data and therefore were unable to measure the effect of diabetes-related treatments, which have been shown to affect pancreatic cancer risk [31]. It is possible that this may account for some of the attenuation in risk seen by duration, as those with the longest duration of diabetes would likely have received the treatments associated with decreased risk for the longest period of time.

In summary, our results support a growing literature that suggests diabetes is an independent risk factor for pancreatic cancer. With the rise of the obesity epidemic, the number of diabetics in the United States and globally is increasing rapidly. There are few identified risk factors for pancreatic cancer and even fewer that can be modified. Reducing diabetes by controlling obesity could benefit pancreatic cancer rates, in addition to the many other known health benefits.

\section{Acknowledgments}

The authors thank the investigators from the PanScan Cohort Consortium centers and the study participants, without whom this study would have not been possible. This project has been funded in whole or in part with federal funds from the National Cancer Institute, National Institutes of Health, under Contract No. HHSN261200800001E. The content of this publication does not necessarily reflect the views or policies of the Department of Health and Human Services, nor does mention of trade names, commercial products, or organizations imply endorsement by the U.S. Government. The Alpha-Tocopherol, Beta-Carotene Cancer Prevention Trial was supported by funding provided by the Intramural Research Program of the National Cancer Institute, and the U.S. Public Health Service contracts [(N01-CN-45165, N01-RC-45035, N01-RC-37004]. CLUE II was supported by National Institute of Aging [5U01 AG018033] and National Cancer Institute [CA105069, CA73790]. European Prospective Investigation into Cancer and Nutrition was supported by the European Commission: Public Health and Consumer Protection Directorate 1993-2004; Research Directorate-General 2005; Ligue contre le Cancer; Societé 3 M; Mutuelle Générale de l'Education Nationale; Institut National de la Santé et de la Recherche Médicale (INSERM) (France); German Cancer Aid, German Cancer Research Center, Federal Ministry of Education and Research (Germany); Danish Cancer Society (Denmark); Health Research Fund (FIS) of the Spanish Ministry of Health, The participating regional governments and institutions (Spain); Cancer Research UK, Medical Research Council, Stroke Association, British Heart Foundation, Department of Health, Food Standards Agency, the Wellcome Trust (United Kingdom); Greek Ministry of Health and Social Solidarity, Hellenic Health Foundation and Stavros Niarchos Foundation (Greece); Italian Association for Research on Cancer (AIRC) (Italy); Dutch Ministry of Public Health, Welfare and Sports, Dutch Prevention Funds, LK Research Funds, Dutch ZON (Zorg Onderzoek Nederland) (the Netherlands); Swedish Cancer Society, Swedish Scientific Council, Regional Government of Skane and Västerbotten (Sweden); World Cancer Research Fund (WCRF). The New York University Women's Health Study is supported by the National Cancer Institute research grants [R01CA034588, R01CA098661, P30CA016087] and the National Institute of Environmental Health Sciences Center grant [ES000260]. The Nurses' Health Study is supported by the National Cancer Institute research grants P01CA087969 and R01CA124908. The Health Professional's Follow-up Study is supported by P01CA055075 and R01CA124908. The Physician's Health Study by R01CA097193 and R01CA124908. The Prostate, Lung, Colorectal, Ovarian Cancer Screening Trial was supported by contracts from the National Cancer Institute [University of Colorado Denver, NO1-CN-25514, Georgetown University NO1-CN-25522, Pacific Health Research Institute NO1CN-25515, Henry Ford Health System NO1-CN-25512, University of Minnesota, NO1-CN-25513, Washington University NO1-CN-25516, University of Pittsburgh NO1-CN-25511, University of Utah NO1-CN-25524 Marshfield Clinic Research Foundation NO1-CN-25518, University of Alabama at Birmingham NO1-CN-75022, Westat, Inc. NO1-CN-25476, University of California, Los Angeles NO1-CN-25404]. The PLCO Study is sponsored by National Cancer Institute's Division of Cancer Prevention, in collaboration with the Division of Cancer Epidemiology and Genetics. The Shanghai Men's and Women's Health Studies were supported by the National Cancer Institute extramural research grants [R01CA82729, R01CA70867, R01CA124908] and by the Intramural Research Program of National Cancer Institute (Division of Cancer Epidemiology and Genetics). The Women's Health Initiative is funded by the National Heart, Lung, and Blood Institute through contracts [N01WH22110, 24152, 32100-2, 32105-6, 32108-9, 32111-13, 32115, 32118-32119, 32122, 42107-26, 42129-32, and 44221]. Role of the Sponsors: This project has been funded with federal funds from the National Cancer Institute, National Institutes of Health. The funding agency had no role in the conduct of the study, the interpretation of the data, or the decision to submit the manuscript for publication. 


\section{References}

1. Siegel R, Naishadham D, Jemal A. Cancer statistics. CA Cancer J Clin. 2012; 2012:6210-6229.

2. Parkin DM, Bray F, Ferlay J, Pisani P. Global cancer statistics, 2002. CA Cancer J Clin. 2005; 55(2):74-108. [PubMed: 15761078]

3. Raimondi S, Maisonneuve P, Lowenfels AB. Epidemiology of pancreatic cancer: an overview. Nat Rev Gastroenterol Hepatol. 2009; 6(12):699-708. [PubMed: 19806144]

4. Lynch SM, Vrieling A, Lubin JH, Kraft P, Mendelsohn JB, Hartge P, et al. Cigarette smoking and pancreatic cancer: a pooled analysis from the pancreatic cancer cohort consortium. Am J Epidemiol. 2009; 170(4):403-413. [PubMed: 19561064]

5. Jacobs EJ, Chanock SJ, Fuchs CS, LaCroix A, McWilliams RR, Steplowski E, et al. Family history of cancer and risk of pancreatic cancer: a pooled analysis from the Pancreatic Cancer Cohort Consortium (PanScan). Int J Cancer. 2010; 127(6):1421-1428. [PubMed: 20049842]

6. Amundadottir L, Kraft P, Stolzenberg-Solomon RZ, Fuchs CS, Petersen GM, Arslan AA, et al. Genome-wide association study identifies variants in the ABO locus associated with susceptibility to pancreatic cancer. Nat Genet. 2009; 41(9):986-990. [PubMed: 19648918]

7. Wolpin BM, Kraft P, Gross M, Helzlsouer K, Bueno-de-Mesquita HB, Steplowski E, et al. Pancreatic cancer risk and $\mathrm{ABO}$ blood group alleles: results from the Pancreatic Cancer Cohort Consortium. Cancer Res. 2010; 70(3):1015-1023. [PubMed: 20103627]

8. Wolpin BM, Kraft P, Xu M, Steplowski E, Olsson ML, Arslan AA, et al. Variant ABO blood group alleles, secretor status, and risk of pancreatic cancer: results from the Pancreatic Cancer Cohort Consortium. Cancer Epidemiol Biomarkers Prev. 2010; 19(12):3140-3149. [PubMed: 20971884]

9. Arslan AA, Helzlsouer KJ, Kooperberg C, Shu X-O, Steplowski E, Bueno-de-Mesquita HB, et al. Anthropometric measures, body mass index, and pancreatic cancer: a pooled analysis from the Pancreatic Cancer Cohort Consortium (PanScan). Arch Intern Med. 2010; 170(9):791-802. [PubMed: 20458087]

10. Vrieling A, Bueno-de-Mesquita HB, Boshuizen HC, Michaud DS, Severinsen MT, Overvad K, et al. Cigarette smoking, environmental tobacco smoke exposure and pancreatic cancer risk in the European Prospective Investigation into Cancer and Nutrition. Int J Cancer. 2010; 126(10):23942403. [PubMed: 19790196]

11. DeFronzo RA, Ferrannini E. Insulin resistance. A multifaceted syndrome responsible for NIDDM, obesity, hypertension, dyslipidemia, and atherosclerotic cardiovascular disease. Diabetes Care. 1991; 14(3):173-194. [PubMed: 2044434]

12. Bao B, Wang Z, Li Y, Kong D, Ali S, Banerjee S, et al. The complexities of obesity, diabetes, and the development and progression of pancreatic cancer. Biochim Biophys Acta. 2011; 1885(2): 135-146. [PubMed: 21129444]

13. Chari ST, Leibson CL, Rabe KG, Timmons LJ, Ransom J, de Andrade M, et al. Pancreatic cancerassociated diabetes mellitus: prevalence and temporal association with diagnosis of cancer. Gastroenterol. 2008; 134(1):95-101.

14. Everhart J, Wright D. Diabetes mellitus as a risk factor for pancreatic cancer. A meta-analysis. JAMA. 1995; 273(20):1605-1609. [PubMed: 7745774]

15. Huxley R, Ansary-Moghaddam A, Berrington de Gonzalez A, Barzi F, Woodward M. Type-II diabetes and pancreatic cancer: a meta-analysis of 36 studies. Br J Cancer. 2005; 92(11):20762083. [PubMed: 15886696]

16. The ATBC Cancer Prevention Study Group. The alphatocopherol, beta-carotene lung cancer prevention study: design, methods, participant characteristics, and compliance. Ann Epidemiol. 1994; 4:1-10. [PubMed: 8205268]

17. Gallicchio L, Chang H, Christo D, Thuita L, Huang H, Strickland P, et al. Single nucleotide polymorphisms in inflammationrelated genes and mortality in a community-based cohort in Washington County, Maryland. Am J Epidemiol. 2008; 167:807-813. [PubMed: 18263601]

18. Calle E, Rodriguez C, Jacobs E, Almon M, Chao A, McCullough M, et al. The American Cancer Society Cancer Prevention Study II Nutrition Cohort: rationale, study design and baseline characteristics. Cancer. 2002; 94:2490-2501. [PubMed: 12015775] 
19. Riboli E, Hunt K, Slimani N, Ferrari P, Norat T, Fahey M, et al. European prospective investigation into cancer and nutrition (EPIC): study populations and data collection. Public Health Nutr. 2002; 5:1113-1124. [PubMed: 12639222]

20. Wolpin BM, Michaud DS, Giovannucci EL, Schernhammer ES, Stampfer MJ, Manson JE, et al. Circulating insulin-like growth factor binding protein-1 and the risk of pancreatic cancer. Cancer Res. 2007; 67(16):7923-7928. [PubMed: 17699799]

21. McWilliams RR, Bamlet WR, Cunningham JM, Goode EL, Andrade Md, Boardman LA, et al. Polymorphisms in DNA repair genes, smoking, and pancreatic adenocarcinoma risk. Cancer Res. 2008; 68(12):4928-4935. [PubMed: 18544627]

22. Zeleniuch-Jacquotte A, Gu Y, Shore RE, Koenig KL, Arslan AA, Kato I, et al. Postmenopausal levels of sex hormones and risk of breast carcinoma in situ: results of a prospective study. Int $\mathrm{J}$ Cancer. 2005; 114(2):323-327. [PubMed: 15540225]

23. Hayes RB, Sigurdson A, Moore L, Peters U, Huang W-Y, Pinsky P, et al. Methods for etiologic and early marker investigations in the PLCO trial. Mutat Res. 2005; 592(1-2):147-154. [PubMed: 16054167]

24. Xu W-H, Zhang X-L, Gao Y-T, Xiang Y-B, Gao L-F, Zheng W, et al. Joint effect of cigarette smoking and alcohol consumption on mortality. Prev Med. 2007; 45(4):313-319. [PubMed: 17628652]

25. Zheng W, Chow W-H, Yang G, Jin F, Rothman N, Blair A, et al. The Shanghai Women's Health study: rationale, study design, and baseline characteristics. Am J Epidemiol. 2005; 162(11):11231131. [PubMed: 16236996]

26. Anderson GL, Manson J, Wallace R, Lund B, Hall D, Davis S, et al. Implementation of the women's health initiative study design. Ann Epidemiol. 2003; 13(9 Suppl 1):S5-S17. [PubMed: 14575938]

27. Rexrode K, Lee I, Cook N, Hennekens C, Buring J. Baseline characteristics of participants in the Women's Health Study. J Womens Health Gend Based Med. 2000; 9(1):19-27. [PubMed: 10718501]

28. Hassan MM, Bondy ML, Wolff RA, Abbruzzese JL, Vauthey J-N, Pisters PW, et al. Risk factors for pancreatic cancer: case-control study. Am J Gastroenterol. 2007; 102(12):2696-2707. [PubMed: 17764494]

29. Jamal MM, Yoon EJ, Vega KJ, Hashemzadeh M, Chang KJ. Diabetes mellitus as a risk factor for gastrointestinal cancer among American veterans. World J Gastroenterol. 2009; 15(42):52745278. [PubMed: 19908334]

30. Chodick G, Heymann AD, Rosenmann L, Green MS, Flash S, Porath A, et al. Diabetes and risk of incident cancer: a large population-based cohort study in Israel. Cancer Causes Control. 2010; 21(6):879-887. [PubMed: 20148361]

31. Li D, Abbruzzese J. New strategies in pancreatic cancer: emerging epidemiologic and therapeutic concepts. Clin Cancer Res. 2010; 16(17):4313-4318. [PubMed: 20647474]

32. Batty G, Kivimaki M, Morrison D, Huxley R, Smith G, Clarke R, et al. Risk factors for pancreatic cancer mortality: extended follow-up of the original Whitehall Study. Cancer Epidemiol Biomarkers Prev. 2009; 18(2):673-675. [PubMed: 19190162]

33. Hemminki K, Li X, Sundquist J, Sundquist K. Risk of cancer following hospitalization for type 2 diabetes. Oncologist. 2010; 15(6):548-555. [PubMed: 20479278]

34. Ammon HPT. Hyper- and hypoinsulinemia in type-2 diabetes: what may be wrong in the secretory mechanism of the B-cell. Exp Clin Endocrinol Diabetes. 1997; 105(S02) 43,7.

35. Gapstur SM, Liu K, Gann PH, Dyer A. Abnormal glucose metabolism and pancreatic cancer mortality. JAMA. 2000; 283(19):2595-2596.

36. Batty GD, Shipley MJ, Marmot M, Smith GD. Diabetes status and post-load plasma glucose concentration in relation to site-specific cancer mortality: findings from the original Whitehall study. Cancer Causes Control. 2004; 15(9):873-881. [PubMed: 15577289]

37. Michaud DS, Wolpin B, Giovannucci E, Liu S, Cochrane B, Manson JE, et al. Prediagnostic plasma C-peptide and pancreatic cancer risk in men and women. Cancer Epidemiol Biomarkers Prev. 2007; 16(10):2101-2109. [PubMed: 17905943] 
38. Stattin P, Björ O, Ferrari P, Lukanova A, Lenner P, Lindahl B, et al. Prospective study of hyperglycemia and cancer risk. Diabetes Care. 2007; 30(3):561-567. [PubMed: 17327321]

39. Grote V, Becker S, Kaaks R. Diabetes mellitus type 2: an independent risk factor for cancer? Exp Clin Endocrinol Diabetes. 2010; 118(1):4-8. [PubMed: 20127570]

40. Grote V, Rohrmann S, Nieters A, Dossus L, Tjønneland A, Halkjær J, et al. Diabetes mellitus, glycated haemoglobin and C-peptide levels in relation to pancreatic cancer risk: a study within the European Prospective Investigation into Cancer and Nutrition (EPIC) cohort. Diabetologia. 2011; 54(12):3037-3046. [PubMed: 21953276]

41. Stolzenberg-Solomon RZ, Graubard BI, Chari S, Limburg P, Taylor PR, Virtamo J, et al. Insulin, glucose, insulin resistance, and pancreatic cancer in male smokers. JAMA. 2005; 294(22):28722878. [PubMed: 16352795]

42. Li D, Yeung S-CJ, Hassan MM, Konopleva M, Abbruzzese JL. Antidiabetic therapies affect risk of pancreatic cancer. Gastroenterol. 2009; 137(2):482-488.

43. McFarland MS, Cripps R. Diabetes mellitus and increased risk of cancer: focus on metformin and the insulin analogs. Pharmacotherapy. 2010; 30(11):1159-1178. [PubMed: 20973689]

44. Pannala R, Leirness JB, Bamlet WR, Basu A, Petersen GM, Chari ST. Prevalence and clinical profile of pancreatic cancer-associated diabetes mellitus. Gastroenterol. 2008; 134(4):981-987.

45. Yachida S, Jones S, Bozic I, Antal T, Leary R, Fu B, et al. Distant metastasis occurs late during the genetic evolution of pancreatic cancer. Nature. 2010; 467(7319):1114-1117. [PubMed: 20981102]

46. World Cancer Research Fund (WCRF) and American Institute for Cancer Research (AICR). Food, nutrition, physical activity, and the prevention of cancer: a global perspective. Washington, DC: AICR; 2007.

47. Li D, Morris JS, Liu J, Hassan MM, Day RS, Bondy ML, et al. Body mass index and risk, age of onset, and survival in patients with pancreatic cancer. JAMA. 2009; 301(24):2553-2562. [PubMed: 19549972]

48. Genkinger JM, Spiegelman D, Anderson KE, Bernstein L, van den Brandt PA, Calle EE, et al. A pooled analysis of 14 cohort studies of anthropometric factors and pancreatic cancer risk. Int $\mathrm{J}$ Cancer. 2011; 129(7):1708-1717. [PubMed: 21105029]

49. Centers for Disease Control and Prevention. National diabetes fact sheet: national estimates and general information on diabetes and prediabetes in the United States, 2011. Atlanta, GA: US Department of Health and Human Services, Centers for Disease Control and Prevention; 2011.

50. National Institute of Diabetes and Digestive and Kidney Diseases. National Diabetes Statistics, 2007 fact sheet. Bethesda, MD: National Institutes of Health; 2008. 
Population (Cases/Controls)

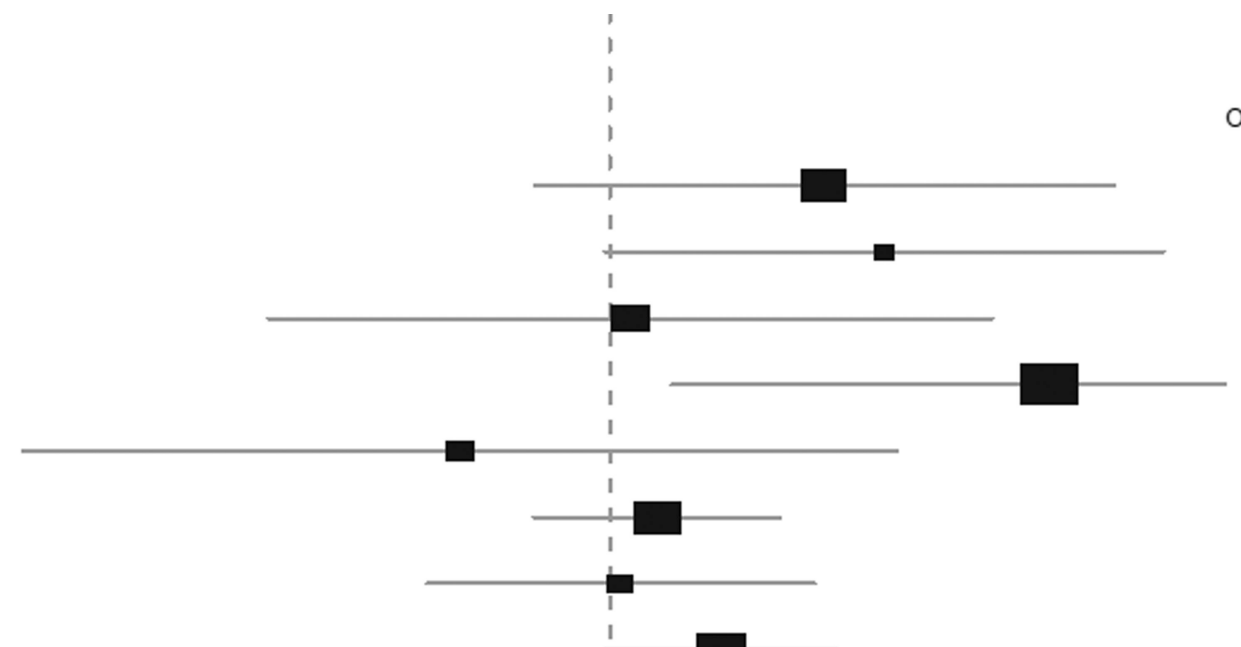

OR $(95 \% \mathrm{Cl})$

CLUE II (43/70)

CPS II (165/165)

EPIC (370/410)

NHS (88/88)

PLCO (243/250)

SMWHS (75/79)

WHI (264/277)

Summary (1467/1563)

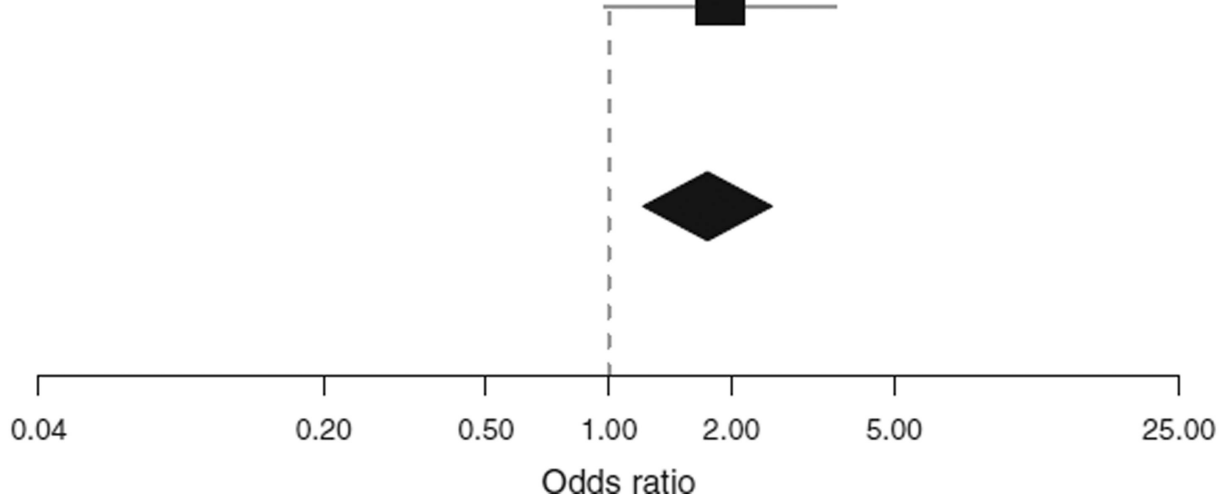

$3.35(0.65-17.2)$

$4.71(0.97-22.8)$

$1.12(0.15-8.67)$

$12.0(1.42-101)$

$0.43(0.04-5.07)$

$1.31(0.65-2.62)$

$1.06(0.36-3.17)$

$1.87(0.98-3.60)$

$1.75(1.21-2.51)$

Fig. 1.

Risk estimates for pancreatic cancer associated with diabetes by study for 2-8 years 
Population (Cases/Controls)

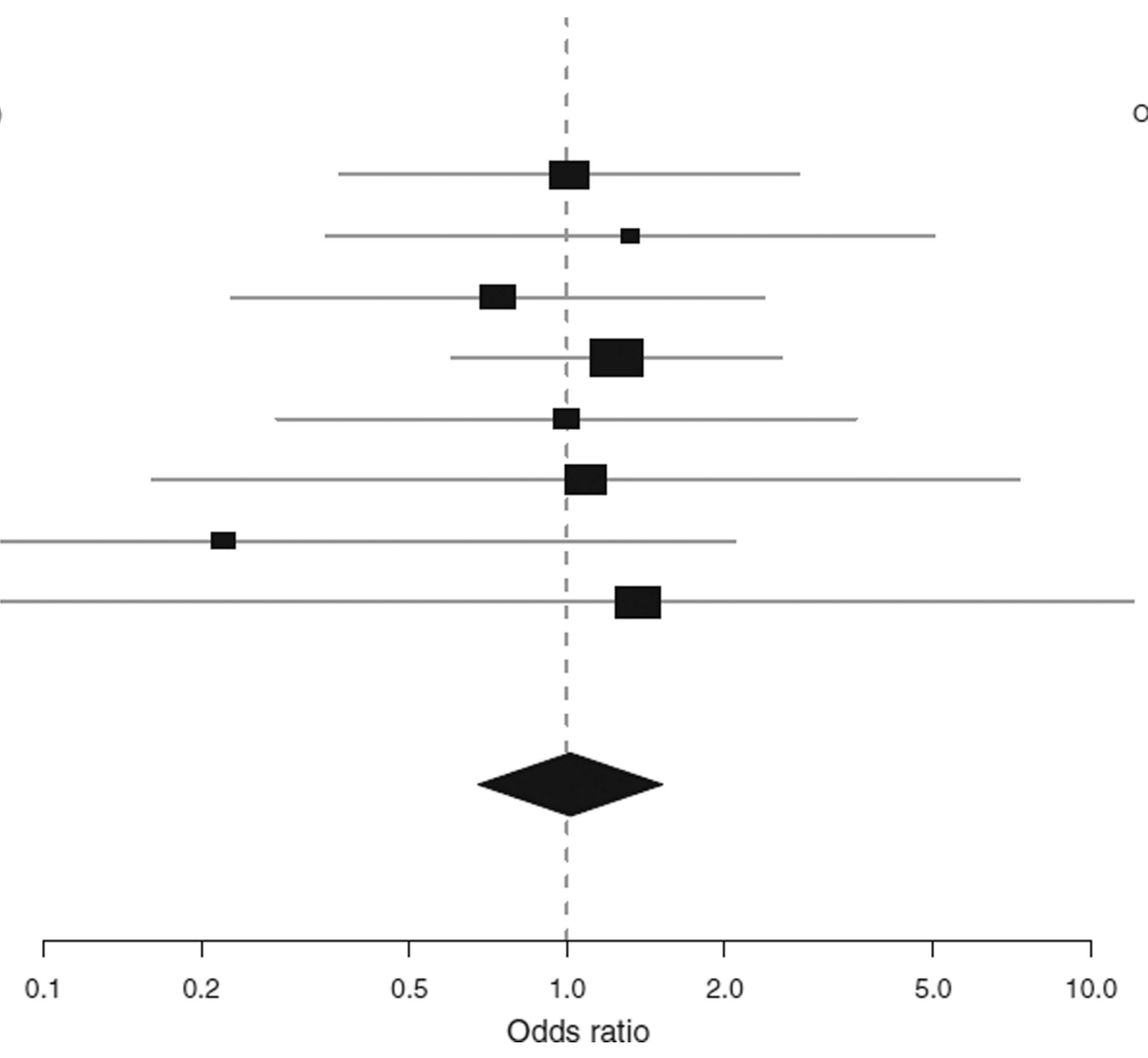

$\mathrm{OR}(95 \% \mathrm{Cl})$

CLUE II (43/70)

CPS II (165/165)

EPIC (370/410)

NHS (88/88)

$\operatorname{PLCO}(243 / 250)$

SMWHS (75/79)

WHI (264/277)

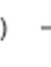

Summary (1467/1563)

$1.01(0.68-1.52)$

Fig. 2.

Risk estimates for pancreatic cancer associated with diabetes by study for 9+ years 


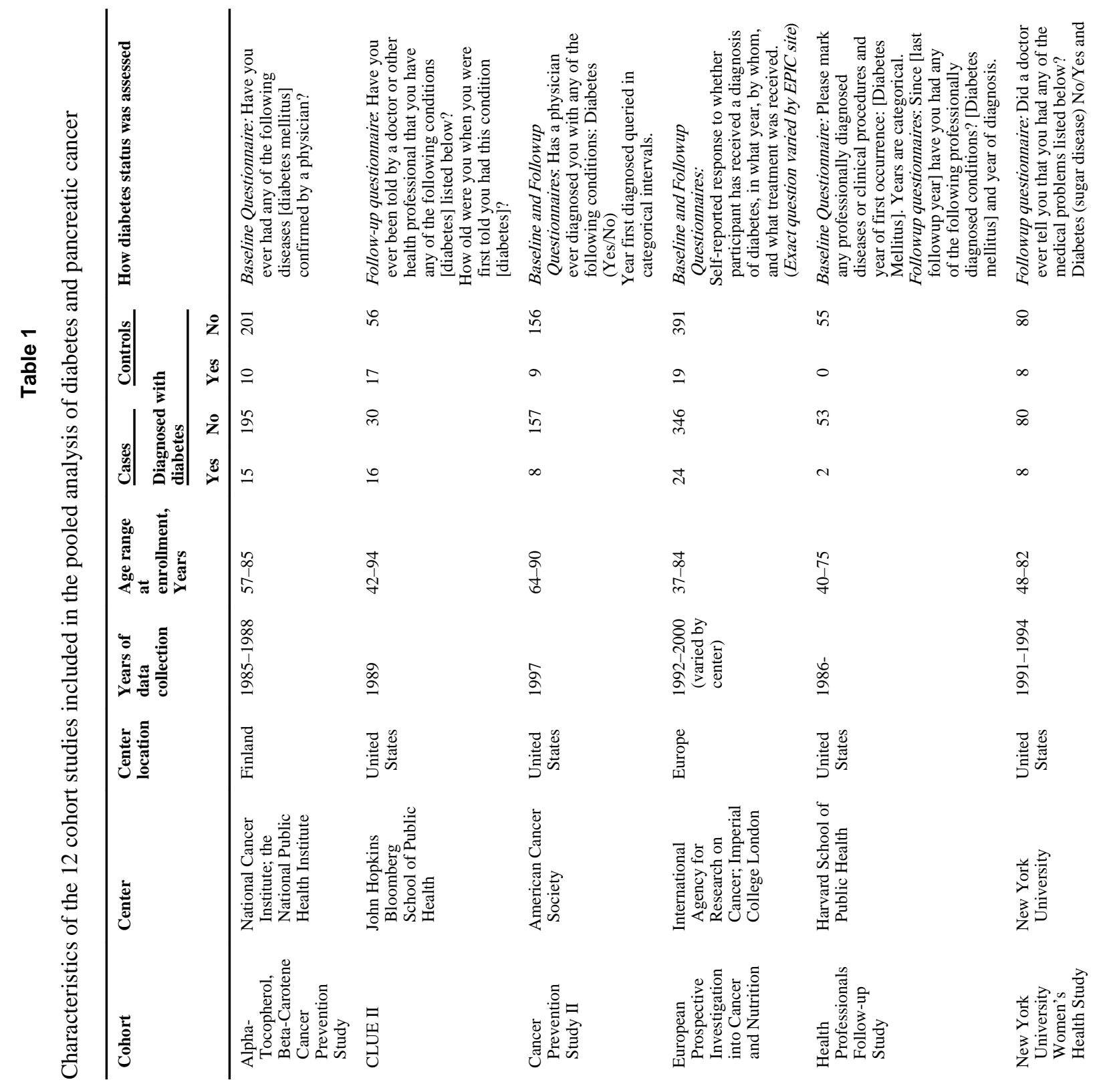




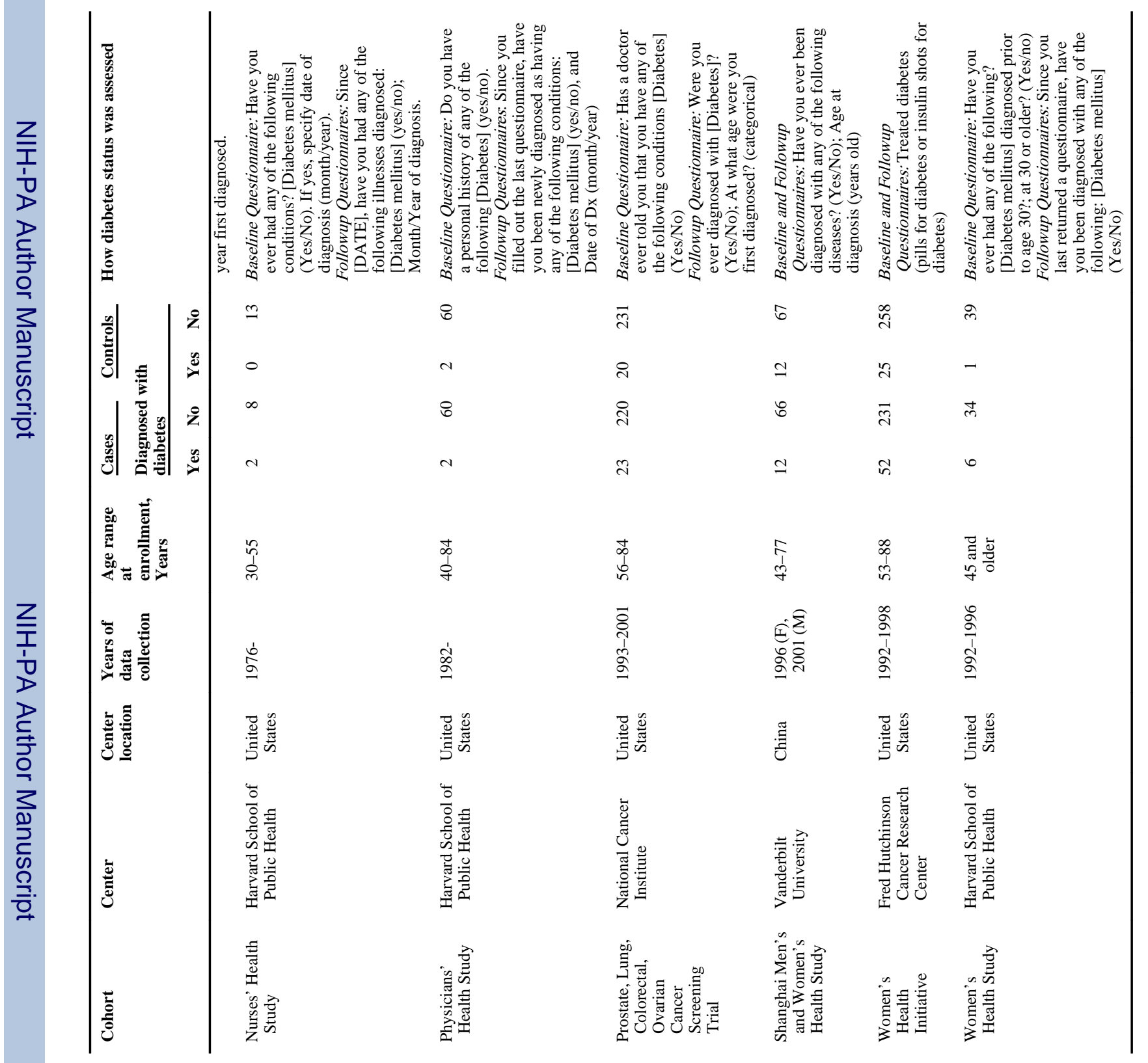


Table 2

Selected baseline characteristics of those included in the pancreatic cancer cohort consortium

\begin{tabular}{|c|c|c|c|c|}
\hline & \multicolumn{2}{|l|}{ Cases } & \multicolumn{2}{|l|}{ Controls } \\
\hline & Number & $\%$ & Number & $\%$ \\
\hline \multicolumn{5}{|c|}{ Age at baseline (years) } \\
\hline$\leq 50$ & 41 & 2.9 & 25 & 1.5 \\
\hline $51-60$ & 213 & 12.9 & 184 & 10.6 \\
\hline $61-70$ & 652 & 39.5 & 708 & 40.9 \\
\hline $71-80$ & 613 & 37.2 & 692 & 40.0 \\
\hline $81+$ & 131 & 7.9 & 121 & 7.0 \\
\hline \multicolumn{5}{|l|}{ Gender } \\
\hline Male & 783 & 47.5 & 816 & 47.2 \\
\hline Female & 867 & 52.6 & 914 & 52.8 \\
\hline \multicolumn{5}{|l|}{ Ancestral race } \\
\hline European & 1,465 & 88.8 & 1,574 & 91.0 \\
\hline African & 32 & 1.9 & 33 & 1.9 \\
\hline Asian & 102 & 6.2 & 105 & 6.1 \\
\hline Other/Unknown & 51 & 3.1 & 18 & 1.0 \\
\hline \multicolumn{5}{|l|}{ Smoking status } \\
\hline Non-smoker & 638 & 38.7 & 730 & 42.2 \\
\hline Former smoker & 551 & 33.4 & 611 & 35.3 \\
\hline Current smoker & 455 & 27.6 & 381 & 22.0 \\
\hline Unknown & 6 & 0.4 & 8 & 0.5 \\
\hline \multicolumn{5}{|c|}{ Body mass index $\left(\mathrm{kg} / \mathrm{m}^{2}\right)$ at baseline } \\
\hline$<18.5$ & 15 & 0.9 & 20 & 1.2 \\
\hline $18.5-<2$ & 623 & 37.8 & 726 & 42.0 \\
\hline $25-<30$ & 676 & 41.0 & 657 & 38.0 \\
\hline $30+$ & 329 & 19.9 & 322 & 18.6 \\
\hline Missing & 7 & 0.4 & 5 & 0.3 \\
\hline \multicolumn{5}{|c|}{ Personal history of diabetes } \\
\hline Yes & 170 & 10.3 & 123 & 7.1 \\
\hline No & 1,480 & 89.7 & 1,607 & 92.9 \\
\hline \multicolumn{5}{|c|}{ Personal history of pancreatitis } \\
\hline Yes & 21 & 1.3 & 2 & 0.1 \\
\hline No & 550 & 33.3 & 565 & 32.7 \\
\hline Missing & 1,079 & 65.4 & 1,163 & 67.2 \\
\hline \multicolumn{5}{|c|}{ Family history of pancreatic cancer } \\
\hline Yes & 44 & 2.7 & 25 & 1.5 \\
\hline No & 729 & 44.8 & 760 & 43.9 \\
\hline Missing & 877 & 53.2 & 945 & 54.6 \\
\hline
\end{tabular}

Table includes all participants with diabetes information, including those diagnosed with diabetes less than 2 years before pancreatic cancer diagnosis or selection as a control $(n=36)$ and also any participants diagnosed with diabetes after cancer/selection $(n=4)$ 
Table 4

Pancreatic cancer risk by duration of diabetes

\begin{tabular}{lrrrl}
\hline Population & Case & Control & Total & OR $^{\boldsymbol{a}}(\mathbf{9 5} \% \mathbf{C I})$ \\
\hline All participants & & & & \\
Never & 1,480 & 1,607 & 3,087 & Ref \\
<2 Years & 29 & 11 & 40 & $3.02(1.47,6.21)$ \\
2-3 Years & 11 & 11 & 22 & $1.10(0.46,2.62)$ \\
3-4 Years & 27 & 10 & 37 & $2.97(1.41,6.26)$ \\
5 Years & 15 & 11 & 26 & $1.42(0.64,3.16)$ \\
6 Years & 17 & 11 & 28 & $1.66(0.76,3.63)$ \\
7-8 Years & 21 & 13 & 34 & $1.95(0.96,3.96)$ \\
9-11 Years & 23 & 17 & 40 & $1.44(0.76,2.74)$ \\
12-15 Years & 11 & 17 & 28 & $0.74(0.34,1.61)$ \\
16+ Years & 16 & 22 & 38 & $0.89(0.46,1.74)$ \\
\hline
\end{tabular}

${ }^{a}$ Adjusted by age, race, gender, study, smoking, alcohol use, body mass index, and family history of pancreatic cancer 